\title{
Gute Stimmung, neue Perspektiven
}

\author{
Armin Wöhrle
}

\begin{abstract}
Auf dem ersten Vier-Länder-Kongress diskutierten Experten aus Hochschulen und Forschungseinrichtungen den Stand und die Entwicklung von Sozialwirtschaft und Sozialmanagement. In drei Schwerpunkten ging es um Studien und Konzepte für die Ausbildung, um Forschung und Entwicklung sowie um die Theoriebildung. Ein Ergebnis der Fachtagung ist die angestrebte Internationalisierung der Bundesarbeitsgemeinschaft Sozialmanagement/Sozialwirtschaft.
\end{abstract}

Nachdem seit einiger Zeit fast alle Bereiche der Sozialwirtschaft beständig Wachstumsraten aufweisen, boomen nun auch die Studiengänge, mit denen für die Sozialwirtschaft und für das Sozialmanagement ausgebildet wird. Nach neuesten Untersuchungen von Karl-Heinz Boeßenecker und Andreas Markert (2007) haben wir es heute im deutschsprachigen Raum mit annähernd hundert Studiengängen zu tun, in denen mindestens 200 Professuren eingerichtet worden sein müssen, viele andere Professuren eingebunden sind und eine Vielzahl an Lehrbeauftragten mitwirken. Es dürften circa 2.000 Absolventinnen und Absolventen diese Studiengänge jährlich mit wissenschaftlichen Arbeiten (immerhin eine ganze Reihe von Masterarbeiten und auch einige Doktorarbeiten) verlassen. Auch das wissenschaftliche Lehrpersonal und die Professoren und Professorinnen legen mit Sicherheit nicht wenige wissenschaftliche Arbeiten vor. Es muss also eine Menge Material zusammengekommen sein.

Gegenwärtig mehren sich die Anzeichen, dass die Zeit der lediglichen Ansammlung von anwendungsbezogenem Material zu Ende geht und umschlägt in eine Phase der Sich- tung, Sortierung und Verortung. Es deutet sich an, dass etwas Eigenständiges aus diesem boomenden Bereich auch im Hinblick auf die theoretische Verarbeitung erwächst. Ausdruck für die Erstellung von Übersicht und Komprimierung auf Wesentliches ist das 2008 von Bernd Maelicke herausgegebene erste Lexikon der Sozialwirtschaft. Im Zusammenhang damit entstanden erste Beiträge mit Überlegungen zur Theoriebildung (vgl. Wendt/Wöhrle 2007). Mit dem VierLänder-Kongress vom 24. bis 26. April 2008 in Luzern wurde eine weitere systematische Bestandsaufnahme zur Ausbildung, Forschung und Entwicklung sowie hinsichtlich der Theoriebildung im deutschsprachigen Raum zu schaffen gesucht.

Einmalig war an diesem Kongress nicht nur das Ansinnen, einen Überblick über Studiengänge, Forschung und Entwicklung sowie Theoriebildung im deutschsprachigen Raum zu schaffen, einmalig war auch die dezidierte Ausrichtung auf Hochschulvertreter und Hochschulvertreterinnen. Waren alle bisherigen Tagungen, Kongresse und Messen praxisorientiert und auf unmittelbar verwertbares Wissen aus, so stand hier die theoretische Bestandsaufnahme im Vordergrund und was daraus zu folgern ist. Bei einer derartigen Ausrichtung kann nicht mit Tausenden von Teilnehmenden gerechnet werden wie bei der ConSozial. Eher erstaunlich war deshalb eine Hundertschaft an Besuchern und Besucherinnen und 26 Beiträge aus der Schweiz, Österreich und Deutschland. Auch Liechtenstein war dabei, Südtirol fehlte leider.

In Pausengesprächen, aber auch in Beiträgen sowie in der Auswertung der Veranstalter wurde immer wieder die angenehme Atmosphäre betont. Zu ihr hat sicherlich das hervorragende Kongressmanagement an der Hoch-

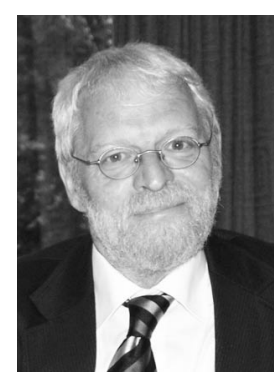

Prof. Dr. Armin Wöhrle ist Industriekaufmann, DiplomSozialpädagoge (FH) und Diplom-Pädagoge. Er hat langjährige Erfahrung als Organisationsberater, Fortbildner und Supervisor. Als Hochschullehrer für Sozialmanagement ist er auch im Leitungsbereich der Hochschule Mittweida tätig. Er ist Mitglied im erweiterten Vorstand der Bundesarbeitsgemeinschaft Sozialmanagement/ Sozialwirtschaft. sowie im Beirat für die Zeitschrift SOZIALwirtschaft und für den Informationsdienst SOZIALwirtschaft aktuell. E-Mailwoehrle@htwm.de

schule Luzern unter der Leitung von Herbert Bürgisser beigetragen. Aber sicherlich war es auch die Situation unter so vielen ausgewiesenen Fachleuten auf hohem Niveau theoretische Anliegen diskutieren zu können, die in anwendungsbezogen ausgerichtete Tagungen keinen Platz haben. Wenn sonst das Zusammentreffen so vieler Professoren und Professorinnen eher zu Spannungen aufgrund von Konkurrenzen führt, so war hier der Umgang erstaunlich entspannt, von einer wertschätzenden Haltung und vom Interesse an den Ansätzen in den anderen Ländern geprägt.

Die fachlichen Ergebnisse sind differenziert und können hier nicht umfassend referiert werden. Ich will deshalb die für mich auffälligen Eindrücke skizzieren und daran persönliche Überlegungen anschließen:

- Hinsichtlich der Studiengänge ist für mich erstaunlich, dass sich das Wachstum ungebrochen fortsetzt. Hier sollten Verbleibstudien angestrengt werden, ob und wo die Absolventinnen und Absolventen unterkommen. Registriert werden muss darüber hinaus, dass die Studiengänge nicht nur in Fachbereichen der Sozialen Arbeit angeboten werden. Neben den Wirtschaftswissenschaften und einigen anderen Fachbereichen an staatlichen Hochschulen nehmen in jüngerer Zeit auch Studienangebote an pri- 
vaten Hochschulen zu. Welche Folgen dies hinsichtlich der Berücksichtigung von Standards der Sozialen Arbeit hat, sollte dringend untersucht werden.

- Der Schwerpunkt Forschung und Entwicklung war gut besetzt, dennoch gab er für mich nicht die Breite der notwendigen Forschung und Entwicklung wieder. Anhand einzelner Evaluierungen beispielsweise von Qualitätsmanagementansätzen wurden unbefriedigende Ergebnisse deutlich und ein hoher Bedarf an vertiefender Forschung. Deutlich wurde von den anwesenden Forscherinnen und Forschern wie Geschäftsführern und Geschäftsführerinnen herausgestellt, dass ein großes Defizit hinsichtlich der Investition in Forschung besteht, aber insbesondere die in Entwicklung geradezu desaströs unterbelichtet ist. Träger können hierfür keine Overheadkosten ausbilden, die jedoch unbedingt notwendig sind, um sich hinsichtlich eines europäischen Marktes aufstellen zu können.

- Im Schwerpunkt Theoriebildung wurde insbesondere deutlich, dass die Disziplin der Sozialen Arbeit nicht mehr allein ist hinsichtlich der Theoriebildung zu Sozialwirtschaft und Sozialmanagement. Auffällig war eine geringe Beteiligung von Vertreterinnen und Vertretern mit speziellen Aussagen zum Sozialmanagement. Insbesondere die Vertreterinnen und Vertreter aus dem universitären Spektrum aus Deutschland glänzten mit Abwesenheit. Es wurde deutlich, dass speziell in der Schweiz - durch Forschungsaufträge untersetzt - viel systematischer an der Erforschung des Sozialmanagements gearbeitet wird.

Die Ergebnisse des Kongresses können nachgelesen werden in verschiedenen Veröffentlichungen. Der erste Kongressband, der eine Übersicht über alle Beiträge bietet, soll noch in diesem Jahr im Augsburger Ziel-Verlag veröffentlicht werden. Ihm folgen drei weitere Bände zu den jeweiligen Schwerpunkten Ausbildung in Studiengängen der Sozialwirtschaft und des Sozialmanagements, zu Forschung und Entwicklung sowie zur Theoriebildung.

Im Ergebnis war es für mich - und ich denke, dass diese Einschätzung von den meisten Beteiligten geteilt wird - ein gelungener Einstieg in einen Verständigungsprozess unter den Fachleuten, die in Studiengängen der Sozialwirtschaft und des Sozialmanagements lehren und forschen. Es wurden denn auch Folgekongresse vereinbart. Der nächste Schritt wird eine Ausweitung der Bestandsaufnahme auf den weiteren europäischen Raum sein.

Nicht ausreichend ist das gegenwärtige Ergebnis dafür, dass wir bereits von einem zweiten Professionalisierungsschub für die Soziale Arbeit durch das Sozialmanagement sprechen können, der auch bei der Klientel angekommen ist. Aber als Vision eingebracht, kann dieser Impuls durchaus die die Perspektive vorgeben, die über diesem Kongress schwebte.

Als Ausdruck der guten Stimmung auf dem Kongress kann auch gewertet werden, dass eine gemeinsame Organisationsform angestrebt wird. Die Bundesarbeitsgemeinschaft Sozialmanagement/Sozialwirtschaft, die diesen Kongress initiierte und in Kooperation mit der Hochschule Luzern und dem FH Campus Wien realisierte, erklärte sich durch ihren Vorsitzenden Herbert Bassarak und die anwesenden Vorstandsmitglieder bereit, sich hinsichtlich einer Organisation für den deutschsprachigen Raum zu öffnen und entsprechend umzubilden. Die Vertreter und Vertreterinnen aus den anderen deutschsprachigen Ländern und Gebieten verdeutlichten ihr Interesse, keine eigenen Organisationsformen ins Leben rufen zu wollen, sondern mit in dieses neue Konstrukt einzusteigen.

\section{Literatur:}

Boeßenecker, K.-H./Markert, A.: Sozialmanagement studieren. Studienangebote im Bereich Sozialmanagement und Sozialwirtschaft und Analysen veränderter Rahmenbedingungen, Düsseldorf 2007.

Bassarak, H./Wöhrle, A.: Sozialwirtschaft und Sozialmanagement im deutschsprachigen Raum - Bestandsaufnahme und Perspektiven - Ergebnisse des Vier-LänderKongresses vom 24.-26. April 2008 in Luzern, Augsburg 2008.

Maelicke, B. (Hrsg): Lexikon der Sozialwirtschaft, Baden-Baden 2008.

Wendt, R.W./Wöhrle, A.: Sozialwirtschaft und Sozialmanagement in der Entwicklung ihrer Theorie, Augsburg 2007.

\section{Soziales Europa}

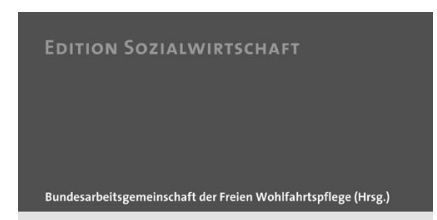

Europa sozial managen Werte - Wettbewerb - Finanzen Bericht über den 5 . Kongress der Sozialwirtschaft vom 26. und 27. April 2007 in Magdeburg

Nomos

Europa sozial managen

Werte - Wettbewerb - Finanzen

Bericht über den 5 . Kongress der Sozialwirtschaft vom 26. und 27. April 2007 in Magdeburg Herausgegeben von der Bundesarbeitsgemeinschaft der Freien Wohlfahrtspflege (BAGFW) 2008, 193 S., brosch., 39,-€, ISBN 978-3-8329-3190-2

(Edition Sozialwirtschaft, $B d .24$ )

Zusammen mit europäischen Partnern können soziale Dienste und Einrichtungen in Deutschland ihre Arbeit optimieren. Die deutsche Sozialwirtschaft kann, wenn sie sich auf internationale Kooperationen einlässt, mit einer europäischen Dividende rechnen. Das war die Botschaft des 5. Kongresses der Sozialwirtschaft 2007 in Magdeburg. Dieser Sammelband dokumentiert Ansprachen, Vorträge, Referate und Präsentationen dieser Veranstaltung.

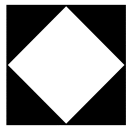

Nomos 